\title{
INVERSE PROBLEMS OF THE INHOMOGENEOUS THEORY OF ELASTICITY FOR THICK-WALLED SHELLS
}

\author{
V. ANDREEV \\ Department of Strength of Materials, Moscow State University of Civil Engineering, Russia.
}

\begin{abstract}
The inhomogeneous theory of elasticity considers bodies, the mechanical characteristics of which (the modulus of elasticity and Poisson's ratio) are functions of the coordinates. If indirect problems of the inhomogeneous theory of elasticity are identified, and the stress-strain state of the body has well-known functions of mechanical characteristics, the essence of inverse problems is to determine the functions of the inhomogeneity for a given stress state of the body. One of the first solutions to such an inverse problem was published in the work of Lekhnitskii ("Radial distribution of stresses in the wedge and half-plane with variable modulus of elasticity". PMM, XXVI(1), pp. 146-151, 1962). In this article, we consider one-dimensional inverse problems for thick-walled cylindrical and spherical shells that are subjected to internal and external pressures in a non-varying temperature field. The aim of this work is to identify the dependence of the elastic modulus on the radial co-ordinate for which the equivalent stress according to a particular theory of strength will be constant at all points of the body (such structures are called equal stress), or the equivalent stress in all points will be equal to the strength of the material (such structures are called equal strength). For example, the author has proven that the limit loads on resulting equal-strength inhomogeneous shells can be significantly increased.

Keywords: Equal-strength structure, equal-stress structure, inhomogeneity, inverse problem, modulus of elasticity, theory of elasticity, thick-walled shell.
\end{abstract}

\section{INTRODUCTION}

According to the basics of solid mechanics, stresses are smaller in the parts of a body where the material is softer than in the rest of the body. This fact makes it possible to reduce the stress concentration by using a softer material in the areas of concentration. This artificial modification of the material is to some degree similar to the plastic behavior of materials. At the transition to the plastic state, the material itself softens and thus prevents failure in the areas of concentration. In thick-walled shells exposed to the influence of internal or external pressures, maximum stresses occur near the inner surface of the shell and lead to failure in this area when the limit state is being reached. The author gives examples of the calculation of the change in the modulus of elasticity in the shells, where failure should be simultaneous at all points, if considered theoretically.

\section{SOME WORDS ABOUT TERMINOLOGY}

If equivalent stresses corresponding to a particular theory of strength at all points of the body are constant, we call this an equal-stress structure. For example, according to the first theory of strength, the equivalent stress is equal to the maximum normal stress and, in accordance with the third theory of strength, it is equal to the maximum shear stress. The constancy of the equivalent stress does not mean that the construction is an equal-stress structure. Figure 1 shows an extended rod; if the features of the rigid support near the point of the force application are not taken into account, $\sigma=F / A$ is assumed constant. Thus, the rod is an equal-stress structure. 
If the rod is homogeneous, this construction is also equal-strength. However, if the rod is made of different materials (Fig. 1b demonstrates components that have different strength values), this rod will be equal-stress, but not equal-strength.

Another example is given in Fig. 2, which demonstrates a phenomenon that is frequently mentioned in books on the strength of materials. According to the above terminology, this beam is neither equal-stress nor equal-strength because the normal stresses in the beam cross-sections vary with depth.

It is clear enough that only a few structures can be equal-stress, such as, in particular, different types of shells such as membrane shells or thick-walled shells exposed to certain types of loading. In this paper, an attempt is made at developing models of equal-stress and equalstrength thick-walled cylindrical and spherical shells, subjected to internal and external pressures within a non-uniform temperature field.

\section{IDEAS}

Figure 3 shows a cross-section of a thick-walled cylindrical shell, loaded internally by constant pressure $p_{a}$ (Fig. 3a). For the homogeneous material (shown by the dotted lines in Fig. 3b), the stress $\sigma_{\theta}$ reaches its maximum near the inner boundary of the cylinder (Fig. 3c), but for the heterogeneous material (solid line), for which $E=E(r)$ as shown in the diagram,

(a)

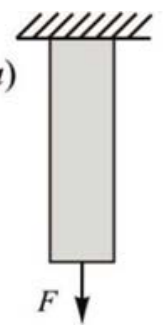

(b)

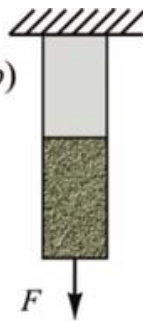

Figure 1: Tension of the rod: (a) equal-strength structure and (b) equal-stress structure.

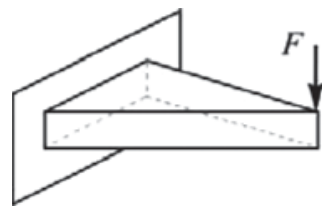

Figure 2: The beam of uniform strength.

(a)

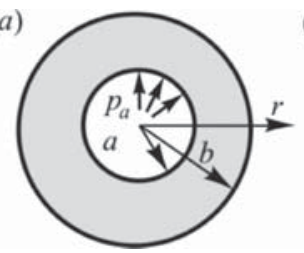

(b)

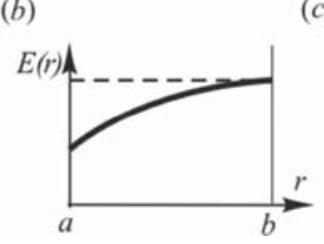

(c)

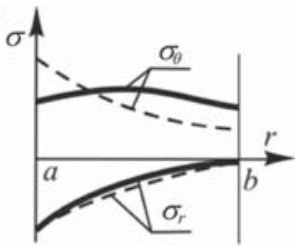

Figure 3: Stress state in a thick-walled cylinder under the action of internal pressure: non-homogeneous material; - --, homogeneous material. 
$\sigma_{\theta}$ remains closer to a constant value. The essence of the method of strength optimization of thick-walled shells consists of developing shells made of heterogeneous materials. For this purpose, the inverse problem of elasticity of inhomogeneous bodies is solved for the shell in question. The inverse problem has the following objective. In accordance with a particular theory of strength, the equivalent stress $\sigma_{0}$ is assumed to be constant at all points of the shell, and it is assumed that an appropriate function $E(r)$ can be defined. Thus, the shell is called an equal-stress shell. The first solutions to inverse problems concerning thick-walled shells can be found in the book by Andreev [1], and optimization of such shells was considered in the works of Andreev and Potekhin [2,3].

4 INVERSE PROBLEMS FOR THICK-WALLED CYLINDRICAL SHELLS

Solutions of inverse problems within the maximum shear theory and the maximum strainenergy theory are now provided.

\subsection{Basic equations}

The direct problem of the theory of elasticity of inhomogeneous bodies with an axial symmetry in cylindrical coordinates is reduced to the differential equation [1]

$$
\sigma_{r}^{\prime \prime}+\left(\frac{3}{r}-\frac{E^{\prime}}{E}\right) \sigma_{r}^{\prime}-\frac{1-v}{r} \cdot \frac{E^{\prime}}{E} \sigma_{r}=0 .
$$

Here, the modulus of elasticity $E=E(r)$. The objective of the direct problem is to determine the stress state if the function $E(r)$ is available. As mentioned above, the purpose of the solution to the inverse problem is to determine the function $E(r)$ for which the equivalent stress $\sigma_{0}$ (which is different for each theory of strength) will be constant all over the structure. An axisymmetric plane-strain problem is considered involving a thick-walled cylindrical shell loaded by constant internal $\left(p_{a}\right)$ and external $\left(p_{b}\right)$ pressures. The inner radius of the shell is equal to $a$, and the outer one is equal to $b$. In this case, the boundary conditions may be represented as

$$
r=a, \sigma_{r}=-p_{a} ; r=b, \sigma_{r}=-p_{b}
$$

\subsection{Maximum shear theory}

The order of principal stresses can be different depending on the nature of the stress $\sigma_{\theta}$. In the case where $\sigma_{\theta}<0$, the principal stresses are defined as follows: $\sigma_{1}=\sigma_{z}=v\left(\sigma_{r}+\sigma_{\theta}\right), \sigma_{2}=\sigma_{r}$ and $\sigma_{3}=\sigma_{\theta}$. With this assumption in mind, the equal-stress condition after some algebra takes the form $\left[(1-k) \sigma_{r}-\sigma_{\theta}\right] /(2-k)=\sigma_{\theta}=$ const where $k=(1-2 v) /(1-v)$.

By expressing $\sigma_{\theta}$ on the basis of the above and by substituting this expression into the equilibrium equation

$$
\frac{\mathrm{d} \sigma_{r}}{\mathrm{~d} r}+\frac{\sigma_{r}-\sigma_{\theta}}{r}=0,
$$

this leads to

$$
\sigma_{r}^{\prime}+k \frac{\sigma_{r}}{r}=-(2-k) \cdot \frac{\sigma_{0}}{r} .
$$


The solution to this differential equation is represented by the function

$$
\sigma_{r}=\frac{A \cdot r^{-k}-(2-k) \sigma_{0}}{k}
$$

The constants $A$ and $\sigma_{0}$ can be found by using the boundary conditions (2):

$$
A=k \cdot \frac{p_{a}-p_{b}}{b^{-k}-a^{-k}} ; \sigma_{0}=\frac{k}{2-k} \cdot \frac{p_{a} b^{-k}-p_{b} a^{-k}}{b^{-k}-a^{-k}} .
$$

By substituting the stress (5) into eqn (1), we obtain a homogeneous linear differential equation for the distribution of the modulus of elasticity:

$$
E^{\prime}-\frac{A}{\sigma_{0}} \cdot r^{-(k+1)} \cdot E=0
$$

Integrating eqn (7) subject to the initial condition $\left(r=a ; E=E_{0}\right)$ leads to the function $E(r)$ :

$$
E(r)=E_{0} \exp \left[-\frac{A}{k \sigma_{0}} \cdot\left(r^{-k}-a^{-k}\right)\right] .
$$

Figure 4 shows the plots of $E(r)$ calculated for the values $v_{1}=0.1, v_{2}=0.25, v_{1}=0.4$, $b / a=2, p_{a}=6 \mathrm{MPa}, p_{a}=12 \mathrm{MPa}$. For the values of the Poisson's ratio $v_{1}=0.1$ and $v_{2}=0.25$, the function $E(r)$ is determined by solving the problem for a cylinder that satisfies the condition $\sigma_{z}>\sigma_{r}>\sigma_{\theta}$, but for the value $v_{3}=0.4$ the solution meets the condition $\sigma_{r}>\sigma_{z}>\sigma_{\theta}$ (details not provided here). As follows from Fig. 4, the influence of the Poisson's ratio on the distribution function $E(r)$ is significant. Figure 5 demonstrates the distribution of stresses in the inhomogeneous (equal-stress) cylinder in the case of $v_{2}=0.25$, as well as in a homogeneous cylinder that has the same dimensions and loads.

Once again, it is pointed out that the equivalent stress $\sigma_{0}$ is the same at all points of the cylinder. Thus, the model of the equal-stress structure has been obtained. However, such a cylinder is not an equal-strength one. The procedure of developing a model of an equalstrength cylinder will be discussed further.

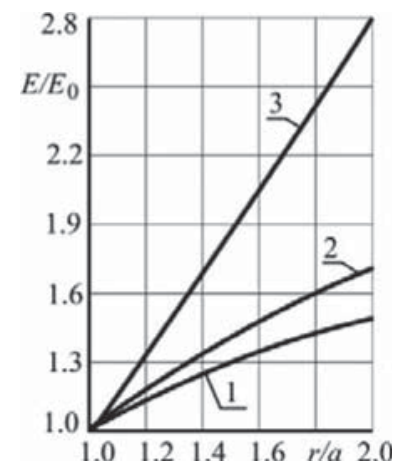

Figure 4: Distribution of the modulus of elasticity in an equal-stress cylinder: $1: v=0.1,2$ : $v=0.25,3: v=0.4$. 


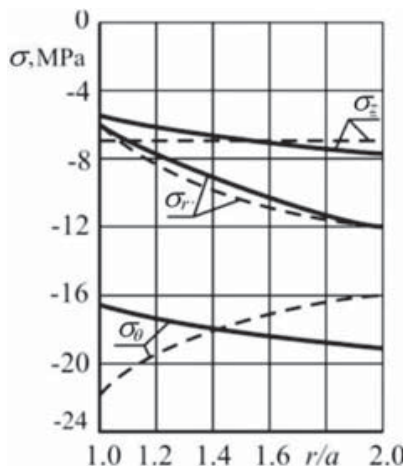

Figure 5: Stress diagrams in an equal-stress cylinder: _- , non-homogeneous material; - - homogeneous material.

Table 1: Mechanical properties of the polymer concrete filled with silica flour.

\begin{tabular}{lccc}
\hline & $\begin{array}{c}\text { Level of filling with silica } \\
\text { flour }\end{array}$ & Prism strength $R_{b}, \mathrm{MPa}$ & $\begin{array}{c}\text { Elasticity modulus } E_{b} \times 10^{-4}, \\
\mathrm{MPa}\end{array}$ \\
\hline 1 & - & 142 & 3.10 \\
2 & 50 & 146 & 4.50 \\
3 & 100 & 160 & 7.10 \\
4 & 200 & 148 & 10.5 \\
5 & 300 & 132 & 13.7 \\
\hline
\end{tabular}

\subsection{Some mechanical properties of concrete}

In order to build a model of an equal-strength structure, it is necessary that, in the course of transformation of the micro-structure of the material, its strength changes at a slower rate than its modulus of elasticity. It may be, for example, a modified cement concrete or a polymer concrete filled with silica flour [4] (see Table 1). It is evident that the strength of the above materials varies slightly while the values of their modulus of elasticity change significantly.

The relation $R=f(e)$ is introduced to ensure the correlation of strength and stiffness properties of the material. It links the strength and deformation characteristics of the material. This function approximates the experimental data for the selected material. By using this relation, the inverse problem can be solved with reference to the model of an equal-strength structure. Nevertheless, the structure may be not equal-stress, but the condition of equal-strength is ensured by the equivalent stress, $\sigma_{0}$ being constant at each point of the body designated to have material strength $R$.

\subsection{The inverse problem of an equal-strength cylinder}

This section describes the method of optimization of a cylindrical polymer concrete thickwalled shell based on the strength criterion developed by Balandin. This condition is confirmed 
experimentally by Genijev and Kissuk [5] for the concrete under all-round non-uniform compression. The Balandin strength condition in the coordinate system of $\sigma_{1}, \sigma_{2}$ and $\sigma_{3}$ represents the surface of a paraboloid of revolution. Given the fact that compressive stresses bear a minus sign, this expression is represented as follows:

$$
\sigma_{1}^{2}+\sigma_{2}^{2}+\sigma_{3}^{2}-\left(\sigma_{1} \sigma_{2}+\sigma_{2} \sigma_{3}+\sigma_{3} \sigma_{1}\right)+\left(R_{b}-R_{b t}\right)\left(\sigma_{1}+\sigma_{2}+\sigma_{3}\right)=R_{b} R_{b t},
$$

where $R_{b}$ is the design strength of concrete subjected to axial compression (prism strength) and $R_{b t}$ is the design strength of concrete subjected to axial tension. Since the concrete works poorly when in tension, it is possible to put $R_{b}=0$ in (9). The application of this assumption substantially simplifies the optimization problem. Given this simplification, expression (9) can be rewritten as

$$
\sigma_{1}^{2}+\sigma_{2}^{2}+\sigma_{3}^{2}-\left(\sigma_{1} \sigma_{2}+\sigma_{2} \sigma_{3}+\sigma_{3} \sigma_{1}\right)+R_{b}\left(\sigma_{1}+\sigma_{2}+\sigma_{3}\right)=0 .
$$

According to Karpenko [6], the Poisson ratio can reach values close to 0.5 with regard to the limit state of the concrete subjected to compressive stresses. Therefore, we take $v=0.5$ for further calculations. Assuming plane-strain conditions in the cylinder, the principal stresses can be determined as follows: $\sigma_{1}=\sigma_{z}=v\left(\sigma_{r}+\sigma_{\theta}\right), \sigma_{2}=\sigma_{r}$ and $\sigma_{3}=\sigma_{\theta}$. With this in mind, the strength condition (10) reads as

$$
0.75\left(\sigma_{r}\right)^{2}-1.5 \sigma_{r} \sigma_{\theta}+0.75\left(\sigma_{\theta}\right)^{2}+1.5 R_{b}\left(\sigma_{r}+\sigma_{\theta}\right)=0 .
$$

Equation (10) describes a parabola in implicit form, so solutions need to pass to a parametrical expression for stresses $\sigma_{r}$ and $\sigma_{\theta}$ [7]:

$$
\sigma_{r}=-R_{b}\left(-0.5 \phi+0.25 \phi^{2}\right), \sigma_{\theta}=-R_{b}\left(-0.5 \phi+0.25 \phi^{2}\right) .
$$

The relationship between the strength and rigidity properties of the material is provided by

$$
R_{b}=\rho+\omega E_{b},
$$

where coefficients $\rho$ and $\omega$ are determined on the basis of the experimental data. When solving the problem, we use the condition that the Poisson ratio $v=0.5$. Therefore, under plane-strain conditions

$$
\varepsilon_{r}=-\varepsilon_{\theta}
$$

Substituting (14) into the condition of compatibility of strain components, we obtain the equation

$$
\frac{\mathrm{d} \varepsilon_{\theta}}{\mathrm{d} r}+2 \frac{\varepsilon_{\theta}}{r}=0 .
$$

The solution of eqn (15) is represented by the expression

$$
\varepsilon_{\theta}=\frac{\varepsilon_{0}}{r^{2}}
$$

where $\varepsilon_{0}$ is unknown.

To obtain the dependence of the modulus of elasticity of concrete $E_{b}$ on the radial coordinate, the expression of strain

$$
\varepsilon_{\theta}=\frac{1}{E_{b}} \cdot\left[(1-v) \sigma_{\theta}-v \sigma_{r}\right]
$$


will be used. When substituting the value of the Poisson ratio $v=0.5$ into (17), the expressions (16), (12) and (13), after transformation, lead to

$$
E_{b}=\frac{\rho(1-2 \phi) r^{3}(\phi)}{4 \varepsilon_{0}-\omega(1-2 \phi) \cdot r^{3}(\phi)} .
$$

By substituting (13) and (18) into eqn (12), the stresses are expressed in terms of parameter $\varphi$ :

$$
\sigma_{r}=\frac{\rho \varepsilon_{0}\left(-5+12 \phi-4 \phi^{2}\right)}{3\left(4 \varepsilon_{0}-\omega(1-2 \phi) \cdot r^{3}(\phi)\right)}, \sigma_{\theta}=\frac{\rho \varepsilon_{0}\left(1-4 \phi^{2}\right)}{3\left(4 \varepsilon_{0}-\omega(1-2 \phi) \cdot r^{3}(\phi)\right)} .
$$

After substituting (19) into the equilibrium eqn (3), we obtain the nonlinear differential equation

$$
\frac{\mathrm{d} r}{\mathrm{~d} \phi}=\frac{2}{3} \frac{8 \varepsilon_{0}(3-2 \phi) r-\omega(1-2 \phi)^{2} r^{4}}{(1-2 \phi)\left(16 \varepsilon_{0}+\omega(1-2 \phi)^{2} r^{3}\right)},
$$

where $\varepsilon_{0}$ is a constant defined by the expression

$$
\varepsilon_{0}=\frac{R_{b}^{(0)}}{4 E_{b}^{(0)}}\left(1-2 \phi_{a}\right) a^{3} .
$$

Here $\varphi_{a}, R_{b}^{(0)}$ and $E_{b}^{(0)}$ are the initial (at $r=a$ ) values of parameter $\varphi$, the design strength of concrete under axial compression and the modulus of elasticity of concrete, respectively. Equation (21) is obtained by substituting the following values into (17):

$$
\begin{gathered}
v=0.5, \varepsilon_{\theta}=\frac{\varepsilon_{0}}{a^{3}}, E_{b}=E_{b}^{(0)}, \\
\sigma_{r}=\frac{R_{b}^{(0)}}{12}\left(-5+12 \phi_{a}-4 \phi_{a}^{2}\right), \sigma_{\theta}=\frac{R_{b}^{(0)}}{12}\left(1-4 \phi_{a}^{2}\right) .
\end{gathered}
$$

The fourth-order method of Runge-Kutta was used to solve eqn (20).

\subsection{Calculation example}

The solution applicable to a thick-walled cylinder and based on the above Paturoyev method and experimental data [4] is presented below.

The calculation was performed on the basis of the following data: $E_{b}^{(0)}=3.1 \times 10^{4} \mathrm{MPa}$, $R_{b}^{(0)}=141.0 \mathrm{MPa}, p_{a} / p_{b}=1.5, b / a=1.6, v=0.5$. To determine the coefficients in formula (13), standard mathematical functions of MathCAD13 were applied and the following values were obtained: $\rho=126.7 \mathrm{MPa}$ and $\omega=4.6 \times 10^{-4}$ for the first three lines of Table 1. Figure 6 shows the approximated relationship for polymer concrete as well as the experimental points for which it is derived.

The solution of (20) is obtained by the Runge-Kutta fourth-order method for the initial value of the argument $\varphi_{a}=-3.071$. The final value of the argument $\varphi_{b}=-2.222$. The values of the pressures produced on the cylinder are $p_{a}=549.1 \mathrm{MPa}$ and $p_{b}=366.0 \mathrm{MPa}$, respectively. The value of pressure $p_{a}$ is derived as follows. The values of constants $\rho$ and $\omega$, the value of parameter $\varphi=\varphi_{a}$, and also the value $r=a$ are substituted into the expression of the normal radial stress (19). As a result, $\sigma_{r}(r=a)=-549.1 \mathrm{MPa}$. By using the first of relations (2), we find the value $p_{a}$ specified above. The pressure $p_{b}$ is equal to $1.5 p_{a}$. 
Stresses $\sigma_{r}, \sigma_{\theta}$ and $\sigma_{z}$ are shown in Fig. 7. The resulting load on the equal-strength cylinder can be compared with the load applied to a homogeneous structure. Due to condition (11), the greatest equivalent stress is reached on the inner surface of the cylinder. For a homogeneous structure, the value of the internal pressure $p_{a}^{\text {hom }}$ can be found according to the formula

$$
p_{a}^{\text {hom }}=-\frac{2 R_{b}^{(0)}\left(s_{r}+s_{\theta}\right)}{\left(s_{r}-s_{\theta}\right)^{2}},
$$

where $s_{r}=-1, R_{b}^{(0)}$ is the value of the design strength of the polymer concrete at $r=a$ and $\sigma_{r}=p_{a}^{\text {hom }} s_{r}, \sigma_{\theta}=p_{a}^{\text {hom }} s_{\theta}$. Expression (23) is obtained as follows. The stresses in the homogeneous cylinder are expressed as

$$
\sigma_{r}=p_{a}^{\text {hom }} s_{r}, \sigma_{\theta}=p_{a}^{\text {hom }} s_{\theta},
$$

where functions $s_{r}$ and $s_{\theta}$ are obtained by solving the Lame problem for a thick-walled cylinder under the boundary conditions

$$
r=a, s_{r}=-1 ; r=b, s_{\theta}=-d
$$

Here $d$ is the ratio of the external pressure to the value of the internal pressure. The design strength is assumed to be constant:

$$
R_{b}=R_{b}^{(0)}
$$

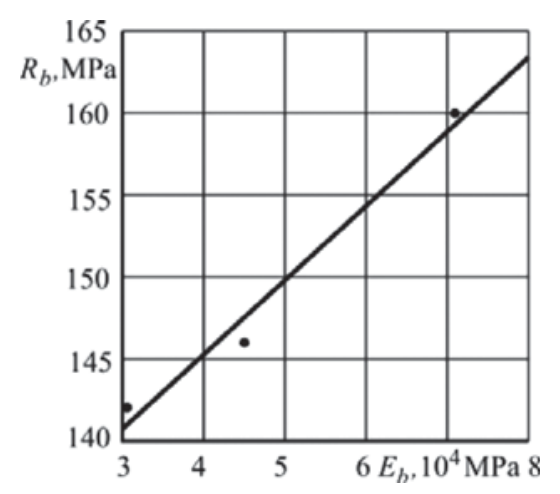

Figure 6: The relationship between strength and modulus of elasticity of the polymer concrete.

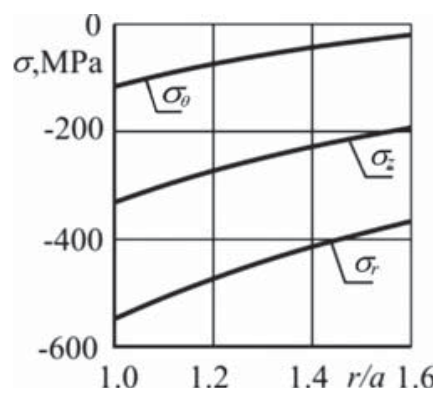

Figure 7: Stresses in the equal-strength polymer concrete cylinder. 
After substituting expressions (24) and (25) into eqn (11), the pressure $p_{a}^{\text {hom }}$ can be found. The calculation performed according to formula (23) gives the value $p_{a}^{\text {hom }}=213.5 \mathrm{MPa}$. Earlier, the limit load for the inhomogeneous equal-strength structure was determined to be $p_{a}^{i n h}=549.1 \mathrm{MPa}$. To determine the effect of the optimized model of the inhomogeneous equal-strength structure, the efficiency ratio $\beta=p_{a}^{i n h} / p_{a}^{\text {hom }}$ is introduced; this indicates how external loads produced on a body can be increased compared with the homogeneous analog. For the equal-strength cylinder considered in this section, $\beta=2.57$. Thus, it has been shown that if a heterogeneous equal-strength thick-walled shell is produced, the action of the load produced on it can be greater by more than 2.5 times.

\section{INVERSE PROBLEMS OF THICK-WALLED SPHERICAL SHELLS SUBJECTED TO TEMPERATURE LOADS}

This section contains some solutions to inverse problems of thick-walled spherical shells loaded by the pressures obtained previously [3] and presented by Andreev [8]. In this section, the inverse problem of a spherical shell is considered, where stresses are caused not only by force loads but also by the temperature field. This problem was solved for the cylindrical shell [9], and for the spherical shell [10]. Solutions to inverse problems in the presence of a temperature field have some features that need further consideration.

As for the previous problems of loaded shells, internal and external pressures must satisfy the condition of equilibrium. For example, if a cylindrical shell is subjected to internal pressure, the equilibrium condition for half of a cylinder is (Fig. 8a)

$$
\Sigma Y=0 \Rightarrow 2 \int_{a}^{b} \sigma_{\theta} \mathrm{d} r=\int_{0}^{\pi} p_{\grave{a}} \sin \theta \cdot \grave{a} \mathrm{~d} \theta=2 \partial_{\grave{a}} \cdot \grave{a} .
$$

If the thick-walled shell is in a steady-state temperature field caused by the temperature difference between the inner and outer surfaces, the stress diagram $\sigma_{\theta}$ is self-balanced (Fig. 8b) and, therefore,

$$
\int_{a}^{b} \sigma_{\theta} \mathrm{d} r=0
$$

For an inhomogeneous cylinder to comply with the condition $\sigma_{\theta}=$ const, it follows from eqn (28) that $\sigma_{\theta} \equiv 0$. Thus, the solution to the inverse problem of a thick-walled shell subjected solely to a temperature field is impossible in the absence of simultaneous action by force loads, including internal and (or) external pressures.

Here the elasticity theory of inhomogeneous bodies is applied to the inverse problem for thick-walled spherical shells under central symmetry. Constant equivalent stresses are
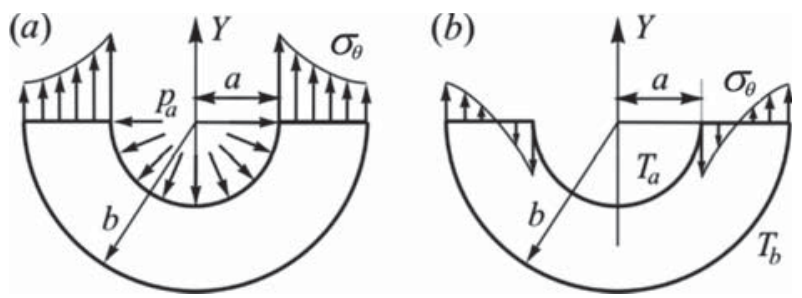

Figure 8: Condition of equilibrium of half of a cylinder: (a) stress diagram $\sigma_{\theta}$ caused by the action of internal pressure; and (b) stress diagram $\sigma_{\theta}$ caused by the action of the temperature field. 
assumed according to the third theory of strength (maximum shear stress theory). It is also assumed that $v=$ const $=0.2$ and $E=E(r)$. The basic notation in this section is the same as used earlier in this paper.

In [1], there is an equation formulated for stress $\sigma_{r}$, which represents the axisymmetric problem of a radially inhomogeneous body:

$$
\sigma_{r}^{\prime \prime}+\phi(r) \sigma_{r}^{\prime}+\psi(r) \sigma_{r}=f(r)
$$

where

$$
\phi(r)=\frac{4}{r}-\frac{E^{\prime}}{E}, \psi(r)=-\frac{2}{r} \frac{(1-2 v)}{(1-v)} \frac{E^{\prime}}{E}, f(r)=-\frac{2 E \varepsilon_{i n}^{\prime}}{r(1-v)} .
$$

Here $\varepsilon_{i n}$ is the induced (in this case temperature) strain, which is computed using the formula

$$
\varepsilon_{i n}=\alpha_{T} T(r),
$$

where $\alpha_{T}=$ const is the temperature coefficient of linear expansion.

Substituting (30) and (31) into (29) leads to the equation

$$
\sigma_{r}^{\prime \prime}+\left(\frac{4}{r}-\frac{E^{\prime}}{E}\right) \sigma_{r}^{\prime}-\frac{2}{r} \frac{(1-2 v)}{(1-v)} \frac{E^{\prime}}{E} \sigma_{r}=-\frac{2 E \alpha_{T} T^{\prime}}{r(1-v)} .
$$

For a steady-state temperature field in a hollow sphere, the inner boundary of which is maintained at temperature $T_{a}=T_{0}$, while the external at $T_{a}=0$, the through thickness temperature is given by the formula

$$
T(r)=T_{0} \frac{a}{a-b}\left(1-\frac{b}{r}\right) .
$$

The boundary conditions for function $\sigma_{r}$ are given in (2). Subsequently, for the purpose of the calculations, it will be assumed that $a=1 \mathrm{~m}, b=2 \mathrm{~m}, p_{a}=0, p_{b}=100 \mathrm{MPa}, E_{0}=2 \times 10^{4}$ $\mathrm{MPa}, v=0.2, T_{a}=100^{\circ} \mathrm{C}$ and $\alpha_{T}=1 \times 10^{-5} 1 /{ }^{\circ} \mathrm{C}$.

Based on the maximum shear stress theory, the relation $E=E(r)$ is determined where the stress state satisfies the condition $\tau_{\max }=\left(\sigma_{\theta}-\sigma_{r}\right) / 2=$ const. Substituting $\sigma_{\theta}-\sigma_{r}=\sigma_{0}=$ const into the equation of equilibrium

$$
\frac{\mathrm{d} \sigma_{r}}{\mathrm{~d} r}+2 \frac{\sigma_{r}-\sigma_{\theta}}{r}=0
$$

gives

$$
\sigma_{r}^{\prime}=2 \frac{\sigma_{0}}{r}
$$

The solution to this differential equation is the function

$$
\sigma_{r}=2 \sigma_{0} \ln \left(\frac{r}{a}\right)+A \text {. }
$$

On the basis of boundary conditions (2), constants $A$ and $\sigma_{0}$ can be determined to be

$$
\mathrm{A}=0, \sigma_{0}=-72.13 \mathrm{MPa} \text {. }
$$


By substituting relation (36) into eqn (32), after some transformations, the first-order differential equation for function $E(r)$ is obtained as

$$
E^{\prime}-\frac{3}{r\left[1+2 k \ln \left(\frac{r}{a}\right)\right]} E-\frac{B}{\sigma_{0} r^{2}\left[1+2 k \ln \left(\frac{r}{a}\right)\right]} E^{2}=0,
$$

where $B$ and $k$ are identified as follows:

$$
B=\alpha_{T} T_{0}(a b) /(1-v)(a-b), \mathrm{k}=(1-2 v) /(1-v) .
$$

Equation (37) is a Bernoulli equation; therefore, for a given Poisson's ratio $v=0.2$, the desired relation $E(r)$ is obtained:

$$
E=\frac{r \sigma_{0}\left[2+3 \ln \left(\frac{r}{a}\right)\right]^{2}}{10 B+6 B \ln \left(\frac{r}{a}\right)+C r \sigma_{0}} .
$$

Constant $C$ can be determined for two possible boundary conditions, that is, the value of Young's modulus $E_{0}$ can be provided at the inner or the outer surface of the shell:

$$
1: r=a, E=E_{0} ; 2: r=b, E=E_{0}
$$

Figure 9 demonstrates the results corresponding to the two options as diagrams of the relationship $E(r)$. It is noteworthy that the quantitative change of the modulus of elasticity, which is the ratio $E(b) / E(a)$, for the two options of the initial condition (39) is almost the same.

Figure 10 shows the distribution of stresses $\sigma_{r}$ and $\sigma_{\theta}$, and Fig. 11 presents the diagram of the maximum shear stress $\tau_{\max }=\left(\sigma_{\theta}-\sigma_{r}\right) / 2$. The results of the calculation of stresses were also the same with respect to both options (39).

By analogy with the test conditions of equilibrium (27), the same test may be performed in the case of a half sphere. Projecting the pressure and stress $\sigma_{\theta}$ onto axis $Z$ (Fig. 12) leads to

$$
\int_{a}^{b} \int_{0}^{2 \pi} \sigma_{\theta} \mathrm{d} F_{1}-\int_{0}^{2 \pi} \int_{0}^{\pi / 2} p_{b} \cos \theta \mathrm{d} F=0
$$

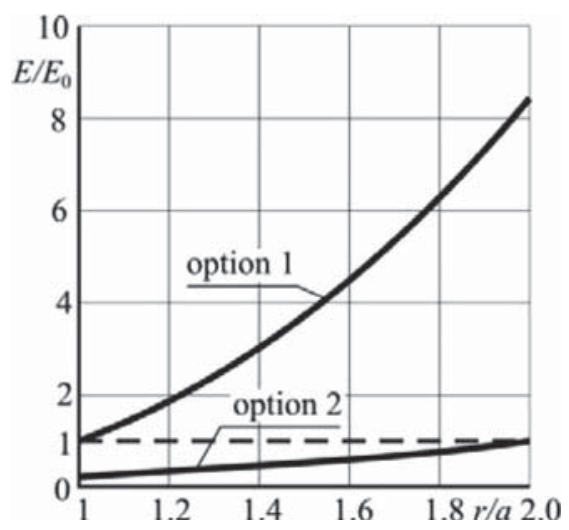

Figure 9: Dependences of the modulus of elasticity in the whole sphere: __, inhomogeneous material; - - -, homogeneous material. 


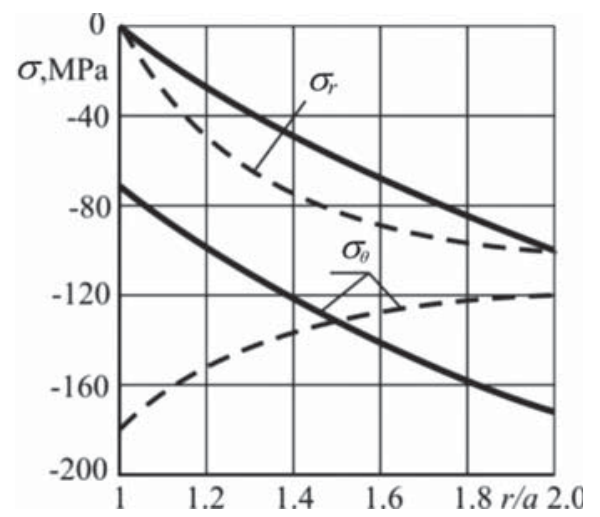

Figure 10: Distribution of normal stressesalong the radius: —_, inhomogeneous material; - - - homogeneous material.

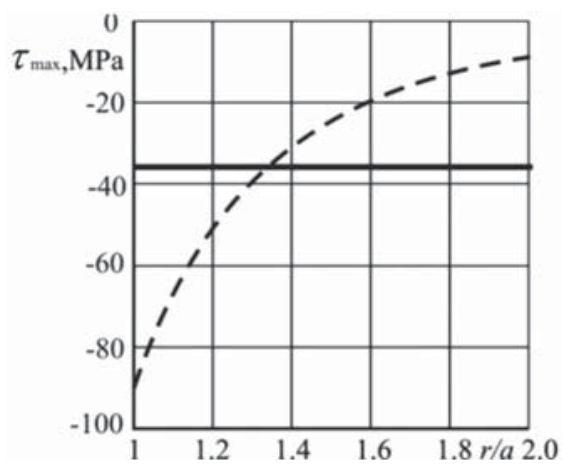

Figure 11: Distribution of maximum shear stress along the radius: — material; - - homogeneous material.

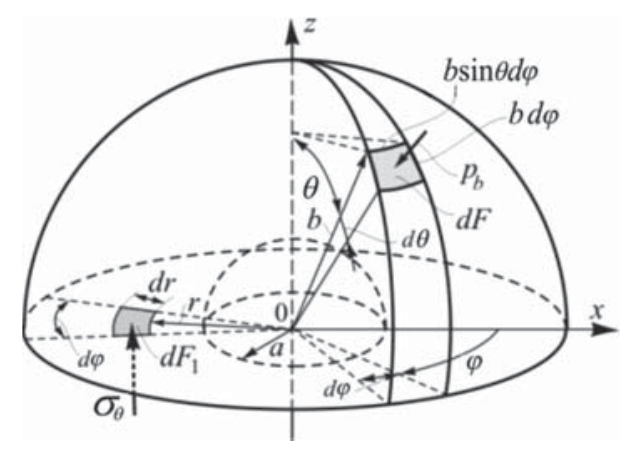

Figure 12: Checking the static equilibrium of the hemisphere. 
which can be re-written as

$$
\int_{a}^{b} \int_{0}^{2 \pi} \sigma_{\theta} r \mathrm{~d} r \mathrm{~d} \phi \sigma_{r}^{\prime}=\int_{0}^{2 \pi} \int_{0}^{\pi / 2} p_{b} \cos \theta \cdot b d \theta \cdot b \sin \theta \mathrm{d} \phi .
$$

The static test (40) has also demonstrated the validity of the results.

It is noteworthy that the maximum shear stress $\left|\tau_{\max }\right|=36.07 \mathrm{MPa}$ in the inhomogeneous equal stress shell, while in a homogeneous sphere $\left|\tau_{\max }\right|=90 \mathrm{MPa}$. Thus, the maximum shear stress in an inhomogeneous shell is about 2.5 times smaller than in the same uniform shell.

The transition to the equal-strength structural model shell can be performed using the method described in subsection 4.4 of this article.

\section{FROM MODELING TO PRACTICE}

It is quite difficult to develop a thick-walled cylinder in which the modulus of elasticity varies along the radius according to a continuous law. Some years ago there was an attempt at designing a cylinder made of epoxy resin EDT-10 filled with quartz sand of different volume fractions. An uncured mixture was poured into a special form, which was placed into a heating chamber for curing and rotating. It was expected that silica particles of different fractions would be distributed continuously along the radius and a material with a variable modulus of elasticity (Fig. 13a) would be obtained. However, after curing, it turned out that all the particles were gathered in a narrow layer near the outer surface of the cylinder, and the result was a two-layer structure (Fig. 13b).

One way of solving the direct problems of the theory of elasticity for inhomogeneous bodies is to replace the continuous function $E(r)$ with a piecewise-homogeneous function. By analogy, it is proposed that optimized thick-walled cylinders consisting of several layers are designed. Thus, the modulus of elasticity of the material of each layer is determined by solving the above inverse problem in which the continuous function $E(r)$ is defined.

The solution to the above problem for a multilayered cylinder is rather simple. It corresponds to the solution of the Lame problem for each layer; therefore, identification of constant values of the boundary conditions (2) and the conditions of an ideal layer-to-layer boundary contact are employed:

$$
u_{i}=u_{i+1} ; \sigma_{r, i}=\sigma_{r, i+1},
$$

where $i$ is the layer number and $u$ the radial displacement.

One of the fundamental questions is how to choose the value of $E_{i}$ in the $i$ th layer. As has been proven by the analysis of the thick-walled cylinder, in order to satisfy the condition of strength (10) at all points of the layer, it is necessary that the $E_{i}$ are equal to the value of $E(r)$ at the left edge of the layer.

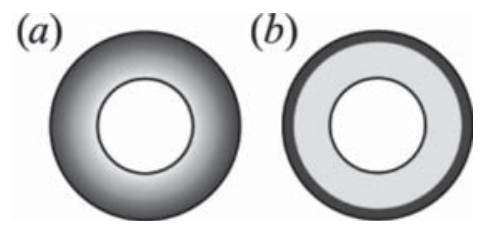

Figure 13: Attempt to design a variable stiffness cylinder: (a) anticipated distribution of quartz sand and (b) resulting distribution of quartz sand. 
Stresses $\sigma_{r}, \sigma_{\theta}$ and $\sigma_{z}$ developing in a three-layer cylinder are demonstrated in Fig. 14, and diagrams of equivalent stresses may be found in Fig. 15.

The resulting load applied to the piecewise-homogeneous cylinder can be compared with the load applied to a homogeneous structure. Using (23), the value of the internal pressure can be found to be $p_{a}^{\text {hom }}=213.5 \mathrm{MPa}$. By comparing this pressure with the load applied to the piecewise-homogeneous cylinder $\left(p_{a}=p_{a}^{(3)}=-\sigma_{r}(r=a)=391 \mathrm{MPa}\right)$, the value of the effectiveness ratio $\beta=p_{a}^{(3)} / p_{a}^{\text {hom }}=1.83$ is obtained. Similar calculations performed for a cylinder consisting of four and five layers produce the following results: $\beta=p_{a}^{(4)} / p_{a}^{\text {hom }}=1.99$, $\beta=p_{a}^{(5)} / p_{a}^{\text {hom }}=2.09$. It is obvious that the larger the number of layers in the cylinder, the closer the value of coefficient $\beta$ to the value $\beta=2.57$, which corresponds to the model with continuous inhomogeneity. Thus, multi-layer structures can be called close to equal-strength structures.

\section{CONCLUSIONS}

In this paper, a summarized method of identification of radial changes in the modulus of elasticity is provided when the equivalent stress in a thick-walled cylinder and a sphere at each point is equal to the strength of the material. The method is based on the solution to the inverse problem of the elasticity theory of inhomogeneous bodies. Thus, designs are equalstrength structures. Since development of such structures is hard enough to implement in practice, it is proposed that multi-layer structures are constructed in which the modulus of elasticity of each layer is identified by solving the inverse problem. Such structures are close to equal-strength structures.

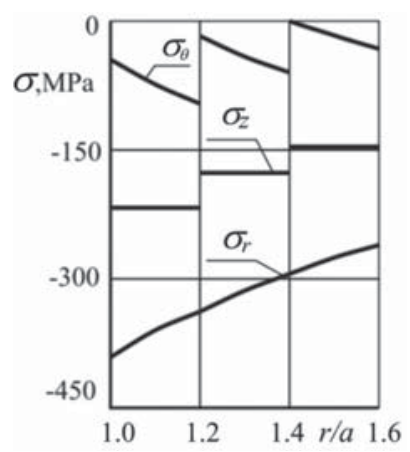

Figure 14: Stress distribution in a three-layer cylinder.

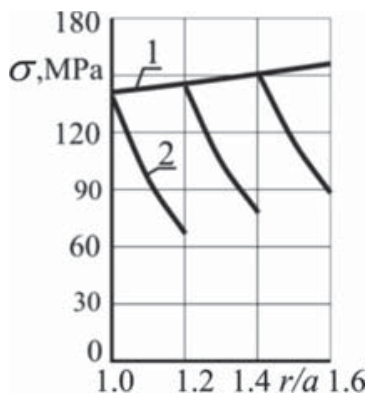

Figure 15: Strength $R_{b}(1)$ and equivalent stress (2) in a three-layer cylinder. 
Models of the above structures are more efficient than those of uniform structures. For example, the value of an external load applied to an inhomogeneous concrete cylinder may be increased almost 2.5 times. Due to the difficulties that accompany development of structures with continuous inhomogeneity, the proposed method of creating multi-layered shells is also very efficient.

In the problems considered the shells are loaded by internal and (or) external pressure, and they may also be under a non-uniform temperature field. It is proven that no structure can be optimized if only the temperature field is present, because the variation of $\sigma_{\theta}$ should be self-balanced, and this property anticipates that $\sigma_{\theta} \equiv 0$.

Generally, such shells may be made of various materials (concrete or reinforced concrete, steels, polymers, etc.). Practical application of these shells will result from the collaborative work of specialists in mechanics, chemistry and technology.

\section{REFERENCES}

[1] Andreev, V.I., Nekotoryje zadachi i metody mehaniki neodnorodnyh tel [Some problems and methods of mechanics of nonhomogeneous bodies], Izdat, ASV: Moscow, 286 pp, 2002.

[2] Andreev, V.I. \& Potekhin, I.A., Modelirovanie I sozdanie ravnoprochnogo tsilindra na osnove iteratsionnogo podkhoda [Modeling and creation equal-strength cylinder based on an iterative approach]. International Journal for Computational Civil and Structural Engineering, 4(1), pp. 79-84, 2008.

[3] Andreev, V.I. \& Potekhin, I.A., Optimizatsija po prochnosti tolstostennykh obolochek [Optimization in strength of thick-walled shells], MGSU: Moscow, 86 pp, 2011.

[4] Paturojev, V.V., Polimerbetony [Polymer concretes], Strojizdat: Moscow, 286 pp, 1987.

[5] Genijev, G.A. \& Kissuk, V.N., K voprosu obobshchenija teorii prochnosti betona [Generalization of the concrete strength theory]. Beton I zhelezobeton, 2, pp. 16-19, 1965.

[6] Karpenko N.I., Obshchie modeli mehaniki zhelezobetona [General models of mechanics of reinforced concrete], Strojizdat: Moscow, 416 pp, 1996.

[7] Kamke, E., Differentialgleichungen, akademie verlag: Leipzig, 576 pp, 1959.

[8] Andreev, V.I., Optimization of thick-walled shells based on solutions of inverse problems of the elastic theory for inhomogeneous bodies. Computer Aided Optimum Design in Engineering XII (OPTI XII). WIT Press, pp. 189-201, 2012.

[9] Andreev, V.I. \& Minayeva, A.S., Postroenie na osnove pervoy teorii prochnosti modeli ravnonapriazhonnogo tsilindra podverzhennogo silovym i temperaturnym nagruzkam [Building on the first theory of strength the model of equal stress cylinder subjected to power and thermal loads]. Privolzhskiy zhurnal, 4, pp. 34-39, 2011.

[10] Andreev, V.I. \& Bulushev, S.V., The solution of the return problem for the equal-stress thick-walled spherical shell subject to power and temperature loadings according to the first and third theories of strength, Proceedings of XXI Russian-Slovak-Polish Seminar “Theoretical Foundation of Civil Engineering”, Warszawa, pp. 93-98, 2012. 